\title{
Challenge Problems in Spectrum Engineering and Waveform Diversity
}

\author{
Hugh Griffiths - University College London, UK (Co-chair SET-182) \\ Shannon Blunt - University of Kansas, USA (Co-chair SET-179) \\ Larry Cohen - Naval Research Laboratory, USA (Co-chair SET-182) \\ Laurent Savy - ONERA, France (Co-chair SET-179)
}

\begin{abstract}
We describe and discuss some of the current challenges facing radar that result from continued spectral encroachment, which necessitating enhanced robustness to interference, agile waveform-diverse operation, and greater synergy between the signal processing and the physical radar/environment. Subsequently, specific research topics are suggested in which spectrum engineering and waveform diversity may yield viable solutions. In so doing, this paper also provides an introduction to the special session on radar spectrum engineering and waveform diversity affiliated with NATO Task Groups SET-182 and SET-179, respectively.
\end{abstract}

\section{INTRODUCTION}

The RF electromagnetic spectrum, extending from below 1 $\mathrm{MHz}$ to above $100 \mathrm{GHz}$, represents a precious resource. It is used for a wide range of applications including communications, radio and television broadcasting, radionavigation, and radar. The exorbitant prices generated from the auction of spectrum for mobile telephony bands in several countries vividly demonstrate the increasing monetary value attached to the RF spectrum. All applications have a need for greater bandwidth, yet this resource is strictly finite. All that can be said with certainty is that the problem of spectral congestion will continue to get worse.

In contrast to the rapid growth of spectral demand, a measurement of spectrum occupancy at a given location as a function of direction, frequency, time and polarisation would be likely to show that the instantaneous spectrum occupancy is underutilized. The reason for this apparent contradiction is that existing allocations are rather inefficient. It therefore follows that there is great potential and need for techniques that use spectrum in a more intelligent, adaptive manner.

For the radar application, waveform diversity is a key enabler of this potential. Modern digital technology now allows for the generation of precise, wide-bandwidth waveforms and provides the means to vary them dynamically, potentially on a pulse-by-pulse basis. Combined with digital, element-level control of the antenna array to enable spatial freedom as well as autonomous decision-making ability (i.e. cognitive radar), the prospective enhancement for radar performance, agility, and breadth of new capabilities is tremendous.

Radar functionality improvement notwithstanding, more intelligent spectral regulation is also necessary. It is unrealistic to adopt an overly conservative position whereby no service should ever interfere with another. However, it is likewise unrealistic to presume that all services may simply co-occupy the same spectrum at the same time because these services may perform quite different functions and thereby have very different operating requirements. As such, it is necessary to understand the nature of interference, what can actually be tolerated by particular applications, and to frame the regulatory environment accordingly.

The purpose of this paper is to introduce and explain the nature of the spectrum congestion problem, to identify a number of specific issues that must be addressed, and to suggest areas of research that offer promise. This paper also serves as an introduction to the other papers in this session which reflect a portion of the work currently being undertaken by members of NATO Task Groups SET-179 on "Dynamic Waveform Diversity \& Design" and SET-182 on "Radar Spectrum Engineering \& Management".

\section{THE RF SPECTRUM}

The rapid expansion by the commercial wireless industry into portions of the spectrum formerly reserved exclusively for radar has resulted in an increasingly congested radar spectrum. The primary driver of this expansion is the growing demand for the spectrally gluttonous application of wireless streaming video to mobile devices [1]. As the radar frequency allocation dwindles, spectral crowding and the deleterious effects of out-of-band leakage further compound this severe and growing problem. With more commercial users occupying spectrum previously assigned solely to radar, and guard bands shrinking or even disappearing, the spurious emissions from consumer electronics are causing increased in-band interference to a multitude of airborne and groundbased radars.

The issues of spectrum congestion and encroachment by other services currently arise mainly in the frequency bands below $5 \mathrm{GHz}$ (C-band). In the higher frequency bands the use of bandwidth is still strictly regulated to prevent interference, especially to critical services such as air traffic control and aircraft landing aids. Since its inception, HF radar has competed for spectrum with the primary users of $\mathrm{HF}$ communications and the amateur radio world. Since the late 1970s, the world's communication industries have shown greater interest in the UHF part of the spectrum. In 1979, the 
World Administrative Radio Conference (WARC) decided to downgrade the primacy of radar in portions of the UHF band, specifically $420-430 \mathrm{MHz}$ and $440-450 \mathrm{MHz}$, to secondary status. In the language of spectrum management, downgrading to secondary status means that radars can operate only as long as they do not interfere with primary users. As a result, HF modalities such as Foliage Penetration (FOPEN) [2] and Over-The-Horizon (OTH) [3] radars have had to develop methods for generating emissions with adequate spectral gaps and associated receive processing to attempt to compensate for these gaps.

Over the last decade, the wireless communications industry has lobbied their member nations within the International Telecommunications Union (ITU) to downgrade radar in the $3.4-3.7 \mathrm{GHz}$ band to secondary status as well. Currently, the big competitor for the $3.4-3.7 \mathrm{GHz}$ band is $4 \mathrm{G}$ wireless communications (WiMAX or LTE; though all indications are that the latter standard will dominate). However, radars operate in particular spectral bands due to a variety of tradeoffs according to the specific function the radar serves. The band from 2-4 GHz, otherwise known as S-band, provides a "sweet spot" that enables a single radar to perform multiple functions from the same aperture. As such, the seemingly simple solution of just moving to a higher and less contentious band is not feasible for many radar applications. Thus it will become necessary for radar systems to contend with commercial emissions in adjacent bands and for radars to provide better containment of their own emissions.

Within the radar band allocations, studies are currently underway to assess the impact of stricter spectral masks on radar emissions $[4,5]$. Spectral roll-off rates presently set at $20 \mathrm{~dB} /$ decade, may be significantly increased to 30 or even 40 $\mathrm{dB} /$ decade, thus predicating the need for substantial improvement in the control of spurious out-of-band emissions (Figure 1). Clearly, the degree to which policy is established must be based on viable operating criteria for the radar and all other services within the RF spectrum.
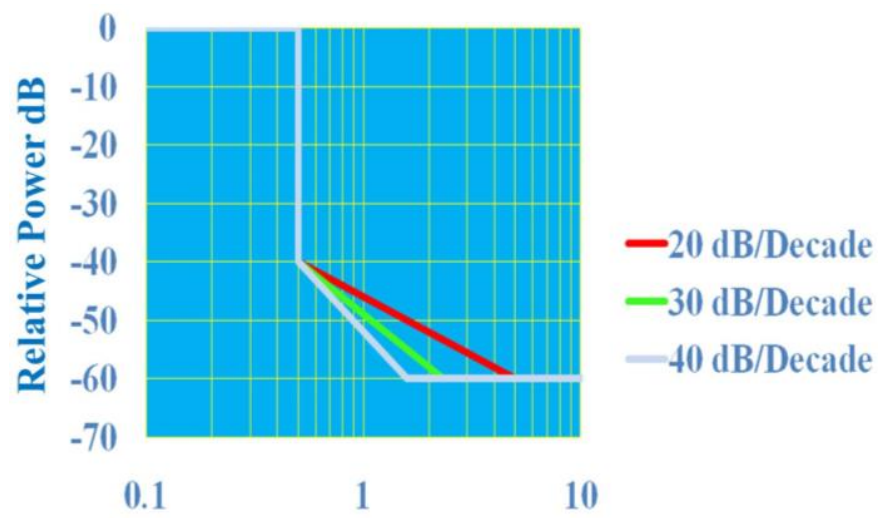

Fraction of the $-40 \mathrm{~dB}$ Bandwidth

Figure 1. Graph of ITU spectral masks being considered (courtesy of John Mettrop, UK Civil Aviation Authority)

\section{TEChNICAL ChallengeS}

There are numerous technical challenges demanding attention due to growing spectral congestion. The ensuing observations and prospective solutions will address pressing needs as well as inform the ongoing policy debate. In light of emerging capabilities promised by waveform diversity some specific challenge problems can be formulated as follows.

\section{A. Expanding Degrees of Freedom}

From a detection standpoint, the ultimate metric for radar is sensitivity, subsuming the notion of discrimination of that which is of interest (target, image, etc.) from the interference and noise. Noise is pervasive and arises from outside the radar (e.g. sky noise) as well as from the components within the radar itself. However, it is interference that tends to be the dominant limiting factor in radar performance. Interference may be intentional (via myriad forms of jamming), self-induced by the radar (i.e. clutter), or unintentional (e.g. spurious emissions or resulting from spectral congestion). To combat the continued increase in both the number and diversity of interference sources, new forms of interference cancellation are required that fully exploit the numerous design freedoms a radar may potentially possess including coding (fast-time), space, slow-time (Doppler), polarization, and frequency.

For example, it is well known that for an airborne radar performing ground moving target indication (GMTI) the clutter becomes coupled in spatial angle and Doppler due to platform motion. As a result, to cancel the clutter on receive it is necessary to operate in a coupled space-time framework, the dimensionality of which is the product of the number of pulse and the number of antenna elements. As the number and breadth of interference sources increases, it is logical to infer that greater dimensionality is needed for interference cancellation. However, unlike the evident angle/Doppler coupling of clutter, it remains an open (and diverse) problem to define how different forms of higher dimensional coupling can be used to contend with increasing interference. The most prominent examples include the various instantiations of multiple-input multiple-output (MIMO) radar and pulse-agile radar that induce different forms of coupling on transmit for subsequent exploitation on receive (e.g. [6,7]). Furthermore, from a pragmatic standpoint it is necessary to address the possible sources of mismatch effects that may arise (e.g. antenna calibration, coherency, etc.) as they produce losses that directly impact sensitivity.

\section{B. Leveraging Wireless Standards}

Beyond just higher dimensionality, knowledge of the radar operating environment should be exploited as much as possible. It is well known that prior knowledge, if sufficiently accurate, can be used to enhance performance for adaptive interference cancellation when operating in 'sample starved' conditions due to data non-stationarity/nonhomogeneity and/or data contamination (i.e. the target selfcancellation effect). From the perspective of spectral congestion driven by wireless communications, it therefore 
stands to reason that prior knowledge about the signal structure of this specific form of interference may potentially be leveraged as a means to better effect its cancellation in the radar receiver. Fortunately, aspects of this signal structure are readily available since commercial wireless communication systems (e.g. WiMAX and LTE) operate according to public standards regarding their emission structure. As these sources of non-intentional interference continue to encroach into radar bands it may be advantageous to develop new forms of interference cancellation that are specifically "tuned" according to these standards.

\section{Passive Sensing \& Multi-Mode Emissions}

The techniques of Passive Bistatic Radar (PBR), exploiting broadcast, communications or radionavigation sources as transmitters of opportunity, have much to offer [8]. It is found that digital transmissions are to be preferred, since their ambiguity functions do not vary with time or with program content, and more closely approach the 'thumbtack' ideal. However, the suppression of the direct signal, multipath and other co-channel interference, which may be more than 100 $\mathrm{dB}$ above thermal noise, represents a particular challenge. Furthermore, the judicious combining of passively sensed echoes from multiple illuminators that possess diverse characteristics in terms of spatial location, directivity, carrier frequency, bandwidth, signal structure (coding), and polarization likewise presents an interesting challenge from a sensor fusion standpoint.

It may also be desirable to design the spectra of such signals so that they not only fulfill their primary purpose but also have favorable properties for radar purposes. This multimode approach has been termed 'commensal' - literally 'at the same table'. Likewise, the design of intentional radar emissions may need to be developed that account for the environment in terms of expectations on clutter properties, intentional/non-intentional interference, and possibly the need to facilitate simultaneously the requirement of other modes such as communications [9], navigation, etc. From the perspective of waveform optimization for a given purpose, it is clearly likely that a commensal approach will necessitate performance trade-offs for the involved modalities. As such, part of the challenge is in determining waveform structures that minimize the performance losses incurred by these tradeoffs.

\section{System-Level Effects}

The signals from radar transmitters are often far from pure; magnetron tubes are particularly poor in this respect. In general, high-power amplifiers (whether tube or solid state) are best operated at a constant power level for reasons of efficiency and 'energy on target'. As such, any theoretical development of waveforms and/or receive processing schemes is incomplete if the non-ideal/nonlinear effects of the radar transmit/receive hardware are not considered. Furthermore, the radar waveform is a continuous signal that is relatively bandlimited (prior to transmitter distortion effects).
Radar components introduce multiple sources of distortion, system losses, and internal noise. Intermodulation products resulting from nonlinear attributes of the system (most notably the power amplifier) and abrupt pulse rise/fall times are known contributors to spectral spreading. Also, the electromagnetic effects imparted by the antenna array (e.g. mutual coupling) and other near-field objects such as the platform and other systems must be considered. Likewise, calibration effects resulting from gain/phase errors across the channels are another source of distortion.

Incorporation of these numerous effects as part of waveform and/or receive processing design is daunting. For the transmit side, one possible approach is to obtain better models for radar system transfer functions (both linear and nonlinear aspects) to use in the development of enhanced transmitter predistortion techniques. The efficacy of such an approach is dependent upon the accuracy of the system model and the degree to which an accurate inverse function can be determined. Alternatively, one could incorporate the transmitter effects into the process for waveform design. This approach intrinsically accounts for distortion-inducing components thereby eliminating the need to 'back out' the attributes of the system. That said the waveform design procedure is now more complicated and becomes specific to particular types of radars (and possibly even individual systems). Furthermore, to meet future requirements on the spectral containment of radar emissions it is anticipated that new transmitter architectures (e.g. [10]) will be needed that likewise may not be conducive to predistortion. New Hardware-in-the-Loop (HiLo) waveform design schemes (e.g. [11, 12]) will therefore be necessary to address this growing complexity.

For the receive side, enhanced sensitivity may be achieved in practice by obtaining greater fidelity. If the physical radar emission is a distorted version of the intended waveform, then a source of mismatch loss is evident. For proposed radar modalities using, for example, pulse agility or MIMO this notion of fidelity-induced loss may become more pronounced due to nonlinear distortion and/or antenna calibration. Beyond the limit of fidelity it becomes necessary to bound the precision with which we can specify a signal structure for advanced receive processing. In other words, in addition to incorporating prior knowledge where possible, we must likewise account for our 'prior ignorance' regarding mathematical models of reality. In some cases this fidelity bound may be expressed as a form of multiplicative noise in signal models, though even this is only a first-order approximation.

\section{E. Co-Design of Transmitter and Waveforms}

As follow-on to addressing transmitter effects there arises the possibility to jointly design the transmitter hardware and associated waveforms [13]. Such a strategy is a departure from traditional design methodology in which either the transmit hardware is fixed with the waveforms modified to suit or a class of waveforms is defined and the system specifications determined to meet the requirements. 
From an optimization standpoint this approach involves multiple, possibly conflicting objectives related to the nature of the physical radar emission and the transmitter topology. Furthermore, the multiple performance trade-offs, nonlinear interactions, and calibration limitations that exist between multiple hardware subsystems, waveforms, and subsequent receive processing yields a rather complicated design problem that may be very difficult to characterize. It therefore becomes necessary to move beyond traditional 'component-specific' effects and characteristics such as waveform/antenna sidelobe levels, bandwidth, beamwidth, intermodulation products, noise figure, frequency stability, insertion loss, quantization noise, isolation, impedance mismatch, mutual coupling etc. and to adopt a comprehensive view of radar operation that incorporates the electromagnetic, systems engineering, and signal processing attributes of the complete system. Such a suggestion is clearly not trivial as the individual 'description languages' (e.g. Maxwell's equations, RF circuitry and system-level operation, and estimation/detection/optimization theory, respectively) are not readily conducive to provide a combined representation. However, the payoff in performance may be well worth the effort.

\section{F. Bio-Inspired Design}

Finally, if one takes many of these challenges to their logical conclusion, what emerges is an RF counterpart to the biosonar capability that has evolved in echo-locating mammals. As we continue to struggle with understanding the precise mechanisms whereby bats/dolphins/whales sense their environment with an astounding degree of accuracy and seemingly rudimentary processing resources [14-17], it is clear that we are still in the very early stages of sensor development.

From the bio-inspired perspective, it is instructive to consider the 'final product' that we observe today in terms of echo-location waveforms and (hypothesized) receive processing as well as attempt to mimic the evolutionary process for RF sensor design. Such (acoustic) waveforms are varied adaptively and dynamically according to the particular phase of the detection/identification/engagement of prey, where the manner in which they are used has been optimized by millions of years of evolution. Such waveforms may take the form of hyperbolic chirps, which are known to possess good Doppler tolerance. They may possess continuous waveform or harmonic components which appear counterintuitive according to our conventional metrics for waveform performance and design. One conjecture is that the harmonic aspect may provide a means with which to distinguish the particular signal of one bat from that of another in the same way as humans have individual voices. In other words, bats may use a form of code-division to operate in a crowded (acoustic) spectrum. All in all, it is clear that our challenge in understanding how these animals can sense their environment so well is only just beginning.

\section{SPeCIAL SESSION OVERVIEW}

Although the spectral congestion problem appears daunting, there are several approaches - both technical and regulatory - that offer some hope of progress. The purpose of the SET-182 Task Group is to study these aspects of the spectral congestion problem and develop various prospective solutions. The SET-179 Task Group is focused on the development of new capabilities that arise from the exploitation of waveform diversity. Because a major focus of waveform diversity research is spectrum related - indeed, spectral concerns were the original impetus for forays into waveform diversity - the two groups are intrinsically linked.

As part of a special session at the 2013 IEEE Radar Conference, this paper also serves as the introduction to the four remaining papers in the session. The paper by Seguin, et al, [18] examines how the frequency shaping effects of an antenna can alter the transmitted waveform. Tigrek and Doyuran [19] consider the Laurent decomposition of the continuous phase modulation (CPM) implementation as a new way in which to frame waveform optimization and subsequent mismatch filtering. In [20] Baylis, et al, describe new methods for joint circuit/waveform design for use in reconfigurable radar transmitters to enhance spectral containment. Finally, Constacias, et al, [21] presents results from experimental trials of the French HYCAM radar that possesses MIMO capability.

\section{CONCLUSIONS}

There are already many examples of problems of mutual interference between radars and other users, and it can be said with confidence that the problem is only ever going to get worse. This paper has concentrated on technical approaches, such as improved transmitter spectral purity, intelligent and cognitive approaches to dynamic frequency allocation, and Passive Bistatic Radar.

The other side of the coin is regulatory: the regulatory framework has thus far taken a relatively conservative approach. However, it is important to have a proper quantitative understanding of the effect of interference of one service upon another in order to adopt appropriate regulation measures, rather than taking the view that no service should ever occupy the same part of the spectrum as any other.

\section{ACKNOWLEDGMENTS}

We acknowledge the contributions of all of our colleagues on the NATO SET-179 and SET-182 Task Groups. We also express our thanks to the various organisations that have funded our work, which include the UK Ministry of Defence, the US Air Force Office of Scientific Research.

\section{REFERENCES}

[1] Cisco Visual Networking Index: Global Mobile Data Traffic Forecast Update, 2012-2017, Feb. 2013.

[2] M.E. Davis, Foliage Penetration Radar, SciTech, 2011. 
[3] G.A. Fabrizio, High Frequency Over-the-Horizon Radar, McGrawHill, 2013.

[4] Manual of Regulations and Procedures for Federal Radio Frequency Management, NTIA, May 2011 Revision of January 2008 Edition.

[5] ITU Manual of Radio Regulations, No.119-08-Rev.1, 6 October 2008.

[6] S.D. Blunt, J. Jakabosky, J. Metcalf, J. Stiles, and B. Himed, "Multiwaveform STAP," IEEE Radar Conf., Ottawa, Canada, Apr./May 2013.

[7] T. Higgins, K. Gerlach, A.K. Shackelford, and S.D. Blunt, "Nonidentical multiple pulse compression and clutter cancellation," IEEE Radar Conf., Kansas City, MO, May 2011.

[8] H.D. Griffiths and C.J. Baker, 'Passive bistatic radar waveforms', and C.J. Baker, H.D. Griffiths and A. Balleri, 'Biologically inspired waveform diversity', (chapters in Waveform Design and Diversity for Advanced Radar Systems (F. Gini, A. De Maio and L. Potter eds) IET, May 2012.

[9] S.D. Blunt, M.R. Cook, and J. Stiles, "Embedding information into radar emissions via waveform implementation," Intl. Waveform Diversity \& Design Conf., Niagara Falls, Canada, Aug., 2010.

[10] F.H. Raab, P. Asbeck, S. Cripps, P.B. Kenington, Z.B. Popovic, N. Pothecary, J.F. Sevic and N.O. Sokal, 'Power amplifiers and transmitters for RF and microwave', IEEE Trans. Microwave Theory \& Techniques, vol. 50, no. 3, Mar. 2002, pp. 814-826.

[11] J. Jakabosky, S.D. Blunt, M.R. Cook, J. Stiles, and S.A. Seguin, 'Transmitter-in-the-loop optimization of physical radar emissions', IEEE Radar Conf., Atlanta, GA, May 2012.

[12] J. Jakabosky, L. Ryan and S Blunt, 'Transmitter-in-the-loop optimization of distorted OFDM radar emissions', IEEE Radar Conf., Ottawa, Canada, Apr./May 2013.

[13] C. Baylis, L. Wang, M. Moldovan, J. Miller, L. Cohen and J. de Graaf, 'Designing transmitters for spectral conformity: power amplifier design issues and strategies', IET Radar, Sonar \& Navigation, Vol. 5, No. 6, pp. 681-685, July 2011.

[14] W.W.L. Au and S.W. Martin, "Why dolphin biosonar performs so well in spite of mediocre 'equipment'," IET Radar, Sonar \& Navigation, vol. 6, no. 6, pp. 566 - 575, July 2012.

[15] G.E. Smith and C.J. Baker, "Echoic flow for autonomous navigation," IET Intl. Radar Conf., Glasgow, UK, Oct. 2012.

[16] J.A. Simmons and J.E. Gaudette, "Biosonar echo processing by frequency-modulated bats," IET Radar, Sonar \& Navigation, vol. 6, no. 6, pp. $556-565$, July 2012.

[17] A. Balleri, H. Griffiths, M. Holderied, and C. Baker, "Bat-inspired multi-harmonic waveforms," Intl. Waveform Diversity and Design Conf., Niagara Falls, Canada, pp. 86-89, Aug. 2010.

[18] S. Seguin, B. Cordill and L. Cohen, 'Radar system impacts due to spectrum attributes of frequency-steerable phased array antennas', IEEE Radar Conf., Ottawa, Canada, Apr./May 2013.

[19] R.F. Tigrek, U.C. Doyuran, 'Utilization of Laurent decomposition for CPM radar waveform design', IEEE Radar Conf., Ottawa, Canada, Apr./May 2013.

[20] C. Baylis, J. Martin, M. Fellows, D. Moon, et al., 'Radar power amplifier circuit and waveform optimization for spectrally confined, reconfigurable radar systems', IEEE Radar Conf., Ottawa, Canada, Apr./May 2013.

[21] L. Constacias, M. Cattenoz, P. Brouard, et al., 'Coherent collocated MIMO radar demonstration for air defence applications', IEEE Radar Conf., Ottawa, Canada, Apr./May 2013. 